\title{
SrS:Eu AND CaS:Eu THIN FILMS: INFLUENCE OF HOST LATTICE ON KINETICS OF EUROPIUM EMISSION*
}

\author{
K. SWIAqTeK, M. GodLEWSKI \\ Institute of Physics, Polish Academy of Sciences \\ Al. Lotników 32/46, 02-668 Warszawa, Poland \\ L. Ninistö AND M. LESKELÄ ${ }^{\dagger}$ \\ Laboratory of Inorganic and Analytical Chemistry \\ Helsinki University of Technology \\ 02150 Espoo, Finland
}

In this paper we report the studies of photoluminescent properties of $\mathrm{CaS}: \mathrm{Eu}$ and SrS:Eu thin films containing up to 3 mole \% of Eu, grown by the atomic layer epitaxy method. The energy transfer and direct intrashell excitation channels of Eu ions are examined in function of temperature.

PACS numbers: $71.55 . \mathrm{Gs}, 76.30 . \mathrm{Kg}, 78.55 . \mathrm{Et}$

\section{Introduction}

Recent studies revealed that rare-earth alkaline earth sulfides may be applied in color thin-film electroluminescent (TFEL) panels [1-6]. From a practical point of view, however, there are still many problems to be solved. The preparation of alkaline-earth sulfide thin films is more difficult than that of $\mathrm{ZnS}$. The EL excitation mechanism in these materials seems to be significantly different from that of conventional Mn doped ZnS TFEL devices [7-12]. In this paper the properties of $\mathrm{Eu}$ activated thin films of $\mathrm{CaS}$ and $\mathrm{SrS}$ are analyzed.

\section{Results and discussion}

The TFEL devices used in the present study were of double insulating type with a $1 \mu \mathrm{m}$ thick phosphor layer of EuS doped (1-3 mole \%) CaS and SrS sandwiched between $0.5 \mu \mathrm{m}$ thick $\mathrm{Ta}_{2} \mathrm{O}_{5}$ insulating layers. The phosphor layers were fabricated by the atomic layer epitaxy method.

*This work was partly supported by the research grant no. 204769101 of the Committee for Scientific Research.

†Dept. of Chemistry, University of Helsinki, 00100 Helsinki, Finland. 
The photoluminescent spectra of the CaS:Eu and SrS:Eu devices measured at $8 \mathrm{~K}$ are shown in Fig. 1. Broad band emissions with peaks at $1.75 \mathrm{eV}$ for $\mathrm{CaS}$ and $1.95 \mathrm{eV}$ for $\mathrm{SrS}$ are due to the $4 f^{6} 5 d^{1}\left(t_{2}\right) \rightarrow 4 f^{7}\left({ }^{8} S_{7 / 2}\right)$ transition of the $\mathrm{Eu}^{2+}$ ion. Contrary to the situation in more ionic crystals doped with Eu ions [10], in CaS and SrS thin films one cannot observe well-separated (in emission or absorption) purely electronic transition. This is related to significant inhomogeneous effects due to disturbed crystal structure of thin films and the high concentration of Eu dopant.

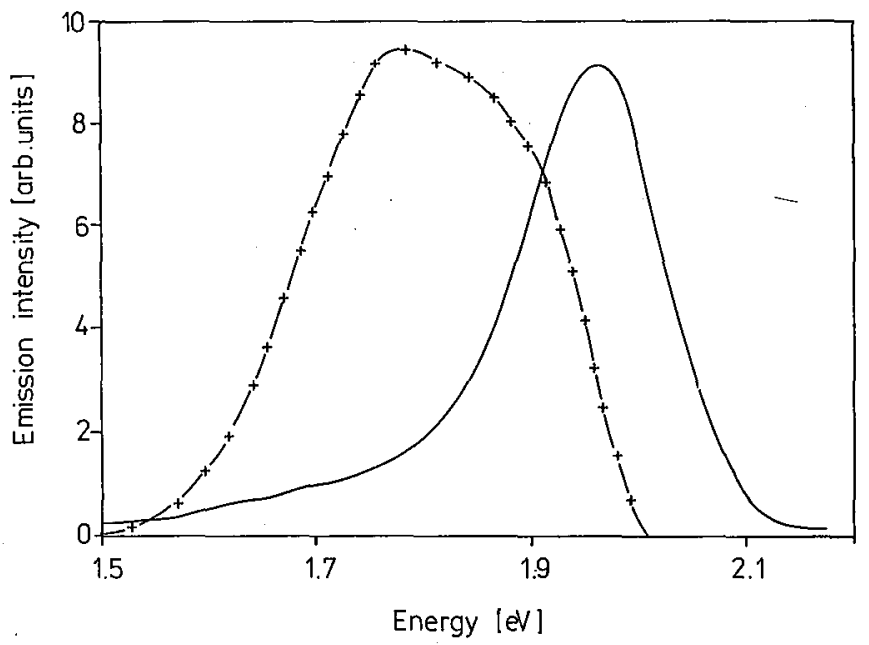

Fig. 1. The photoluminescence spectra of the CaS:Eu (+) and SrS:Eu thin films measured at $8 \mathrm{~K}$ under $488 \mathrm{~nm}$ excitation.

The temperature dependence of the total intensity of $\mathrm{Eu}^{2+}$ emission for both materials is presented in Fig. 2. In both cases, (a) and (b), the emission intensity decreases monotonically with increasing temperature (starting from $8 \mathrm{~K}$ ). However, in the case of SrS:Eu we observe an abrupt increase in intensity for temperatures higher than $200 \mathrm{~K}$. We explain the observed decrease in the emission intensity $I(T)$ by thermal ionization of an electron from the $\mathrm{Eu}^{2+} 4 f^{6} 5 d^{1}$ excited state to the conduction band (CB) of the host lattice. The temperature dependence of the $\mathrm{Eu}^{2+}$ emission in $\mathrm{CaS}: \mathrm{Eu}$ is described with a $39 \mathrm{meV}$ activation energy, whereas the $I(T)$ dependence for $\mathrm{SrS}: \mathrm{Eu}$, in the temperature range 8-200 K, has a two-exponential character with $57 \mathrm{meV}$ and $4 \mathrm{meV}$ activation energies. We tentatively explain the increase in the Eu photoluminescence (PL) in SrS, in the temperature range 200-300 K, by the existence of a deep electron trap, practically inactive at lower temperatures. An electron thermally ionized from this trap to the CB may be captured by the $\mathrm{Eu}^{3+}$ center via the $4 f^{6} 5 d^{1}$ excited state of the $\mathrm{Eu}^{2+}$ ion.

The temperature dependence of the decay kinetics of $\mathrm{Eu}^{2+}$ emission was measured. In the case of the CaS:Eu thin film we observe two, well-separated, decay components with lifetimes in the range of $0.4-0.6 \mu \mathrm{s}$ and $1.4-3.2 \mu \mathrm{s}$, respectively. The temperature dependence of both lifetimes is non-monotonic. After a small 


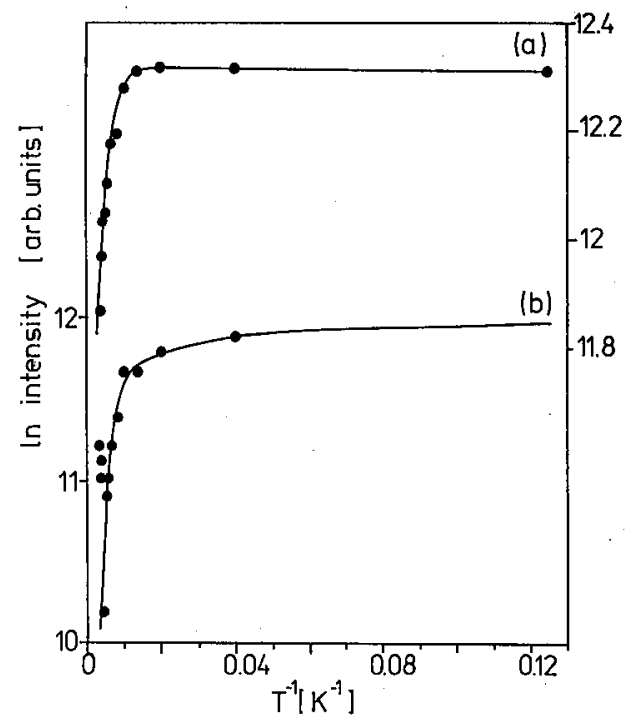

Fig. 2. The temperature dependence of the total emission intensity of $\mathrm{Eu}^{2+}$ ions in $\mathrm{CaS}: \mathrm{Eu}$ (a) and $\mathrm{SrS}: \mathrm{Eu}$ (b) thin films under $488 \mathrm{~nm}$ excitation. From the theoretical fit (solid line) characteristic activation energies were determined.

decrease observed for temperature values increasing from 8 to $100 \mathrm{~K}$, there is a significant increase for temperatures higher than $100 \mathrm{~K}$. Another type of behavior was observed for SrS:Eu thin films. The decay time of the so-called slow component almost linearly increases with increasing temperature. A weak, fast component appears only for the highest temperatures $(300 \mathrm{~K})$.

The obtained results lead to the following conclusions. The temperature dependence of $\mathrm{Eu}^{2+}$ emission intensity in $\mathrm{CaS}: \mathrm{Eu}(3 \mathrm{~mole} \%)$ indicates that thermal activation of electrons to the CB. is controlled by the energy level lying approximately $39 \mathrm{meV}$ below the CB bottom. It is probable that this level is related to the $4 f^{6} 5 d^{1}$ excited state of the $\mathrm{Eu}^{2+}$ impurity. Simultaneously, with an abrupt decrease in the PL intensity near $100 \mathrm{~K}$, there is a significant increase in the decay time in the $\mathrm{Eu}^{2+}$ emission kinetics. It means that some of the electrons in the excited states of the $\mathrm{Eu}^{2+}$ ion recombine radiatively to the $\mathrm{Eu}$ ground state, but the rest of them is thermally activated to the CB. Some of these free electrons may be recaptured by ionized europium centers $\left(\mathrm{Eu}^{3+}\right)$ and they may recombine radiatively via Eu. However, the rest of the electrons in the CB may find other recombination paths leading to a decrease in the Eu PL intensity at high temperatures.

In the case of the SrS:Eu thin films, the situation is more complicated. There are two deactivation energies of the PL intensity ( $57 \mathrm{meV}$ and $4 \mathrm{meV}$ ). The decay time of the Eu emission kinetics slowly and almost linearly rises with increasing temperature. We indicate that this property of $\mathrm{Eu}^{2+} \mathrm{PL}$ is not understood at present and cannot be simply explained by carrier exchange from the excited state 
of the ion to the CB. Several mechanisms considered include Auger type quenching processes, resonant and phonon-assisted energy transfer processes. Additional experiments are necessary to distinguish between these possibilities.

\section{References}

[1] W.A. Barrow, R.E. Coovert, C.N. King, in: 1984 SID Int. Symp. Digest Tech. Papers, 1984, p. 249.

[2] V. Shanker, S. Tanaka, M. Shiiki, H. Deguchi, H. Kobayashi, H. Sasakura, Appl. Phys. Lett. 45, 960 (1984).

[3] S. Tanaka, H. Yoshiyama, Y. Mikami, J. Nishiura, S. Ohshio, H. Deguchi, H. Kobayashi, Appl. Phys. Lett. 50, 119 (1987).

[4] M. Ando, Y.A. Ono, J. Appl. Phys. 68, 3578 (1990).

[5] M. Ando, Y.A. Ono, J. Appl. Phys. 69, 7225 (1991).

[6] M. Ando, Y. Abe, Y.A. Ono, J. Appl. Phys. 70, 5018 (1991).

[7] D.C. Morton, M.R. Miller, E. Schlam, in: Conf. Record 1985 Int. Display Research Conf., 1985, p. 182.

[8] R.S. Crandall, Appl. Phys. Lett. 50, 551 (1987).

[9] R.S. Crandall, Appl. Phys. Lett. 50, 641 (1987).

[10] K. Świątek, M. Godlewski, Appl. Phys. Lett. 56, 2192 (1990).

[11] K. Świątek, M. Godlewski, D. Hommel, Phys. Rev. B 42, 3628 (1990).

[12] K. Świątek, M. Godlewski, D. Hommel, M. Leskelä, L. Niinistö, E. Nykanen, P. Soininen, M. Tiitta, Acta Polytech. Scand. Appl. Phys. Ser. 170, 237 (1990). 